\title{
The Development of Self Help Book for Counseling Guidance's Teacher Experiencing Burnout Symptoms with Counseling Cognitive Behavioral Therapy
}

\author{
Rahmadhania Rizanty ${ }^{\star 1}$ Lara Fridani $^{1}$ \& Happy Karlina Marjo ${ }^{1}$ \\ ${ }^{1}$ Magister of Guidancce and Counseling, State University of Jakarta, Jakarta, Indonesia \\ rahmadhaniarizanty_bk2017@unj.ac.id; lfridani@unj.ac.id; hkarlina@unj.ac.id \\ *Corresponding Author: amadhania21@gmail.com |Phone: +6283829118421
}

\begin{abstract}
Self help book is believed as an alternative method to help someone in order to help himself dealing with the psychological problems that they had experienced. One of the psychological problems which very vulnerable to be experienced by someone that working is emersion of burnout symptoms. The researcher has interviewed Counseling Guidance's (BK) teacher to find the factor that caused emersion of burnout symptoms of work, from the interview some informations has gathered about the causes of Counseling Guidance's teacher experienced burnout symptoms, among others because the number of Counseling Guidance teachers in the school with an unbalanced number of students and unclear duties and roles assigned to Counseling Guidance teachers. Other that, there were many Counseling Guidance teachers did not have an appropriate knowledge with the qualification that have been determined. Every individual has a different level of burnout symptoms and the impact that they experienced was also different. The level of burnout symptoms could be reduced or even eliminated by determining the appropriate method, one of them by counseling approach of Cognitive Behaviour Therapy (CBT) using guided imagery technique.
\end{abstract}

Keywords: counseling teacher; CBT; self help book; burnout symptoms

\section{Introduction}

According to Bergsma, (2008) self help book is an alternative book to help themselves without others, and readers could choose certain themes in self help book, one of the themes is coping stress. In that theme contains the right ways to deal with certain psychological problems. Self help book is made easier for readers to practice the method which avaliable in the book. Robert (2015) explained that self help book usually contains sugestion, course, or certain techniques and fictional stories that link messages and interesting ways.

Psylogical problem that usually experienced someone is work stress until experiencing burnout symptoms. One of the professions that susceptible to feel stress untill burnout symptoms is Counseling Guidance teachers. This is because Counseling Guidance teachers having a lot of demands to help by the right way and mentoring student problems which is quite diverse. Drew et al, (2017) says that sometimes Counseling Guidance teachers would also to be a client because they need a counseling process before they become a professional helper for others. Self help book gives a benefit to the readers who wants to get the information about emotional problems or their psychological experience. Howard (2012) explained that there is a special benefits for self help book readers, such as the readers are able to adapt in particular theme as needed of the problem, practicing the strategies that they learn in the self help book, and for making improvement in life without asking other people helps (professional).

\section{Literature Review}

\subsection{Self Help Book}

Self Help Book is a companion book that used to give the informaion about process help self-recovery. Usually the recovery is related with personal problem and the emotional that caused a negative impact if they do not overcome it immediately. Self help book chosen because relatively easy to get in any condition. Self help book is also cheaper rather than having to go to other professionals and it does not spent much 
time because it could be read anytime.

Self help book is useful to handling early problems rather than there is not professional treatment. National Institute for Health and Clinical Excellence of Raymond et al., (2016) have recommeded self help book as part of way someone intervened of helping someone's problem. Self help book has certain themes that could be customized to your needs, one of them is coping stress. The interview result by Counseling Guidance teachers about method that has been used to reduce level of burnout symptoms could be done by trying to reduce and eliminate burnout symptoms itself, without asking another professional help because it is usually a common thing perceived everyone without exception by Counseling Guidance teachers. Therefore, this research was conducted to make Counseling Guidance teachers easy to get the information about an appropriate way through counseling technique in order to reduce level of burnout that they felt.

\subsection{Burnout Symptoms}

The emergence of burnout symptoms mostly experienced by individual who has dense intensity towards repeated activity. This can be happened to the people who works in social field where their main task is to help other people. Social worker always gets demand and pressure to resolve people's problem as a professional, it is why this kind of professions very susceptible to burnout symptoms. Murai in Lopez (2013) uttered that there are five categories of burnout symptoms. It could be perceived because of physical exhaustion, emotional exhaustion, behavior, and interpersonal. The general symptoms of an individual who has physical exhaustion are trouble of sleeping, headache, flu and digestion problem. Whilst emotional exhaustion are feeling helpless, anxious, easy to get angry, feeling guilty and depression.

Acording to Murai of Lopez (2013) revealed that there are five categories of burout symptoms. Burnout symptoms was due to physical exhaustion, emotional, behavioral, and interpersonal exhaustion. The most common physical symptoms that experienced by someone who feel exhausted are sleeping trouble, headaches, sniffles, flu and digestive problems. Meanwhile, emotional symptoms are feeling of helplessness, anxiety, irritability, guilt and depression.

According to Genç, (2016) there are five levels that usually happen to someone who experienced burnout symptoms. First level, there is dissatisfaction of working, so that it could deplete the energy, lack of time efficiency at work. The second level begins to feel a lack of energy to work, it caused less of sleep till easy to get tired. The third level that they experienced has caused more severe symptoms. This could be seen by the diseases, frequent feelings of anger, and depression. Fourth level is a deep depression marked by severe selfdoubt and being obsessed with personal problems. Fifth level, self-hurting until terminate their career.

The multitude of negative impact of burnout become important to know the main factor which causing burnout according to McCarthy in Mullen \& Gutierrez (2016), is because the demands that challenging as part of the job. The level of stress happened to counselor is caused by the workload that occurs over a long period of time. Affecting to emotional and physical health. Furthermore, the stress will be a bad impact to the Counseling Guidance's teachers performance enabling one's to leave his career. Beside that, according to Wilkerson \& Weldini in Kim \& Lambie (2018) said that there are connection between burnout symptoms' factors with demography where age and experience shows connection with fatigue of work.

Maslach in Padilla et al (2017) explain that there are three general effects of burnout. Firstly, there is a reduction of involvement, all the pleasant things become contrarily, unsatisfactory and meaningless. Secondly, there is a reduction of emotion related to positive feelings, enthusiasm, dedication, enjoying process of work become anger, anxiety and depression. Third, there is an organization problem, from its structure until the process. Moreover, According to Bilot (2012) said that burnout could be harm for someone. There some a negative things because of burnout such as, lose attention, decreasing positive feelings, decreasing feeling of sympathy, lost respect for others. On the other hand, burnout causes cynical attitude, lost of dignity, morale decline, hopelessness, negative attitudes towards work and their self, fatigue and ending up physical illness. This research wants to know burnout symptoms levels of counseling guidance teachers of senior high school and vocational high school in East Jakarta 1 and find out an effective way so that the level of burnout that counseling guidance teachers felt could be reduced and also find out the main cause of the counseling teachers feeling burnout symptoms. According to Maslach which is quoted by Lee, (2018) burnout symptom causing three main things, such as emotional exhaustion (Emotional exhaustion), being personalized (Depersonalization), and also reduced achievement (personal accomplishment).

\subsection{Counseling Cognitive Behavior Therapy (CBT)}

Kabir (2017) explains that CBT is a therapy focusing to one's cognitive. It is widely used to help counseling problem in terms of worry until reducing the level of stress. The benefits of using CBT is counseling process relatively finish in a short time. 
Aclair et al (2018) defines that CBT also has capability to reduce the level of stress. Cognitive therapy generally is short-term therapy (six to twenty sessions) and only focused on very specific problems. CBT focusing of here and now by the problems that appears in daily life.

Kabir (2018) from the Cognitive Behavioral Therapy approach there are relaxation techniques that could decrease stress levels of person, so it is expected to decrease a fatigue consequences of work experienced by someone. Relaxation techniques could be done easily because there are several techniques involved of the relaxation, and those techniques are also could be done by individual or groups. One of the example of relaxation techniques is a guided imagery, a yoga that you can do it by yourself at home. That method is more practical and efficient.

In this relaxation techniques there are many methods that could also decreasing levels of burnout symptoms. According to Rajeswari (2019), a successful techniques for decreasing burnout symptoms is guided imagery. Smeltzer (2002) Guide imagery is a method for relaxation and imagining a place by an incident that related to calmness and pleasant things. On the other hand, imagery techniques used for manage coping by imagining something that started of relaxation process. Generally, it works by asking client to slowly close their eyes and focus on their breath. Client are encouraged to relax, clear and fill their mind with imagination to create peace and calm. Rajeswari,(2019) explained that there is technique that succeeded in reducing the level of burnout symptoms, and that technique is guided imagery.

\section{Materials and Methods}

This study is using type of development research. According to Sugiyono (2011) research and development (Research and Development) is a research method used for research and create a product to test the effectiveness of the product. Frascati (2015) explain that R\&D is a research that intended for the manufacture creative and systematic product to improve the knowledge due to designing a new method from the last one that already exist.

This study using development style Dick and Carey, the development style that comes from the thought is still an empirically tested concept and its implementation is directed started from the planning, implementation to evaluation of results. In the R\&D research conducted by researchers that will be produce a book called self-help book with coping stress theme, in the book there are techniques that customized with certain psychological problems.

In this reasearch, a limited trial will be conducted by 10 counseling teachers which incorporated in MGBK organization.The Research development uses descriptive procedural development which is outlining the steps to be followed to produce a product. The product in this research is media book, where the book contain counseling approaches and techniques used for reducing level of burnout symptoms. Dick, \& Carey, (2015) explained there are development model that consists 10 steps such as (1) identification service purpose, (2) Analysis service, (3) Analysis contexts, (4) determine service purpose, (5) developing instruments assessment, (6) developing service strategy, (7) developing and choosing service material, (8) designing and perform formative valuations, (9) revise, (10) designing and perform evaluate summative. Nevertheless, in this research could only done in the ninth step of revising the self help book so that, it can be used according to the needs of the readers.

\section{Results and Discussions}

The first interviewed was about burnout symptoms to know the cause of someone feels burnout. The result of interview which has done by researchers, could be informed that one of the factors is a double roles passed by counseling guidance teacher. On the other hand, the lack number of counseling guidance teacher made an additional work and not all of other teachers at school was competent in their field and it caused of them felt burnout symptoms.

First demographic data was most of counseling guidance teacher were females about 30-40 years old. The number of senior high school of counseling guidance teacher was more than vocational high school and the duration of work service about 5 to 15 years. The information about need of self help book that has done through questionnaire and shared to counseling guidance teacher of senior high school and vocational high school in east jakarta 1 and has the result that counseling guidance teacher need a self help book to get the information about burnout symptoms and to know the appropriate method on reducing the level of burnout symptoms by using certain technical counseling according to its problem.

As many as 125 of respondents' counseling guidance teacher of senior high school and vocational high school in East Jakarta which contained the instrument Maslach Burnout Inventory-Human Survey Service (MBI-HSS) was related the level of burnout perceived and then processed it using likert scale by three of high, medium, low level categorization in order to know the result of burnout symptoms level of counseling guidance teacher. As much as 125 respondents be discovered that counseling guidance teachers of senior 
high school and vocational high school felt burnout symptoms on high level was 15 respondents. The burnout symptoms on medium level was 15 respondents and 28 respondents for low level. By that result obtained total number of most of the burnout symptoms by counseling guidance teachers of senior high school and vocational high school in east Jakarta at the medium level. if take a look at the total number by three dimensions of high level burnout symptoms was a lack of achievement in the amount of 0,065 next score emotional exhaustion 0f 0,040 and the last pesonalized of 0,090. By that amount score could be seen that burnout symptoms which caused counseling guidance teacher of senior high school and vocational high school in East Jakarta felt lack of achievement at the workplace. Hence, the researcher made a self help book, so that the amount of counseling guidance teachers who experienced amedium level of burnout symptoms could be diminished by the most appropriate method based on the self help book theory to reduce burnout symptoms that they experienced.

In this study, the media that developed is self help book. Self help book itself is an alternative way to make a reader able to know the information about handling psychlogyical problems that they experienced. In the phase of making self help book initiated with choosing media design, there was a colour design, so that reader did not feel surfeited while they read that book. Drafting material made practically in order to make it easy and in that material there is an appropriate counseling approach with the practical technique so that it could be practiced to reduce the level of burnout symptoms that they felt. According to the result of first research that has done, self help book expected easy to get and reachable to all people, therefore the hope of making self help book in order to help counseling guidance teachers and the professioals of the service sector in general, be able to practice the right and appropriate method in order to reduce the level of burnout symptoms that they felt.

\section{Conclusions}

Self help book known as media to help someone resolve the problem which is usually related to psychological. Self help book became one of a special book that has an allure because it contains certain theme that the readers could chose it, one of them is coping stress. In the making of this book, the researcher choose coping stress theme where the book is contain practical ideas in order to escaped or reducing of its sress level.

Feeling stress of counseling guidance teachers inflict a negative impact to optimaize at work. By that matter, if it left by long term would caused a burnout symptoms for someone. Other negative impact of burnout symptoms were cynicism, loss of dignity, decreased morale, feeling of helplessness or hopeless, hopeless towards work, fatigue, and ended up physical disease, for example feeling headache frequently.

As one of counseling approach that has determined to be able to reduce level of burnout symptoms is CBT approach with relaxation technique. Relaxation technique could be done by an easy way because there are some techniques of relaxation, and those techniques could be done individually or group. There some relaxation techniques which could be used to reduce level of burnout symptoms of someone, one of those techniques is guided imagery. Guided imagery technique gives the instruction to someone to be able to practice pleasant things and calming someone mind. If someone has practiced that guided imagery technique expected could reduce burnout symptoms that they felt.

\section{Acknowledgement}

The authors would like to thanks the supervisors from Magister of Guidance and Counseling, State University of Jakarta for their assistance in the completed this study.

\section{Author's Contributions}

All authors discussed the results and contributed to from the start to final manuscript.

\section{Conflict of Interest}

The authors declare that they have no competing interests.

\section{References}

Anclair, M., Lappalainen, R., Muotka, J., \& Hiltunen, A. J. (2018). Cognitive Behavioural Therapy And Mindfulness For Stress And Burnout: A Waiting List Controlled Pilot Study Comparing Treatments For Parents Of Children With Chronic Conditions. Scandinavian Journal of Caring Sciences, 32(1), 389396. https://doi.org/10.1111/scs.12473 
Bergsma, A. (2008). Do self-help books help? Journal of Happiness Studies, 9(3), 341-360. https://doi.org/10.1007/s10902-006-9041-2

Bilot, J. (2012). The Relationship Between Wellness And Burnout Among Noice Counselors. Florida Atlantic University.

Dick, W., Carey, L., \& Carey, J. O. (2015). The Systematic Design of Intruction (Eight). United States of America: Pearson.

Drew, M., Stauffer, M. D., \& Barkley, W. (2017). Personal Counseling in Academic Programs with Counselor Trainees. The Journal of Counselor Preparation and Supervision, 9(1). https://doi.org/10.7729/91.1131

Frascati. (2015). Guidelines for Collecting and Reporting Data on Research and Experimental Development. Directorate for Science, Technology and Innovation, (October), 1-2. Retrieved from https://www.oecd.org/sti/inno/Frascati-Manual-2015-Flyer-EN.pdf

Genç, G. (2016). Learned Resourcefulness and Burnout Levels of English Teachers. International Journal of Psychology and Educational Studies, 3(1), 1-13. https://doi.org/10.17220/ijpes.2016.01.001

Howard. (2012). The Benefits of Reading Self-Help Books. Retrieved from http:/ / www.tyhoward.com/article/The-Benefits-of-Reading-Self-Help-Books

Kabir, S. M. S. (2018). Counseling Approaches. ReseachGate, (March 2017).

Kabir, S. M. S. (2017). Introduction To Counseling. ResearchGate, (March 2017).

Kim, N., \& Lambie, G. W. (2018). Burnout And Implications For Professional School Counselors. The Profesional Counselor, 8(3), 277-294. https://doi.org/10.15241/nk.8.3.277

Lee, D. (2018). Counselors' Self-Discrepancy And Its Impact On Their Burnout And Wellness. University of Arkansas Fayetteville. University of Arkansas.

Lopez, Y. (2013). Factors That Contribute to Burnout Among Elementary School Counselors. Liberty University.

Mullen, P. R., \& Gutierrez, D. (2016). Burnout, Stress, and Direct Student Services Among School Counselors Burnout, Stress and Direct Student Services Among School Counselors, 6, 344-359. https://doi.org/10.15241/pm.6.4.344

Padilla, A. A. G., Benivento, C. V. E., \& Suarez, B. S. P. (2017). Burnout Syndrome and Self-Efficacy Beliefs in Professors. Research Article, 5. https://doi.org/doi.org/10.20511/pyr2017.v5n2.170 ISSN

Rajeswari. (2019). Guided Imagery On Perceived Stress \& Burnout. Naraya Nursing Journal, 5(4). https://doi.org/10.13140/RG.2.2.34579.12320

Raymond, C., Marin, M. F., Hand, A., Sindi, S., Juster, R. P., \& Lupien, S. J. (2016). Salivary Cortisol Levels And Depressive Symptomatology In Consumers And Nonconsumers of Self-Help Books: A pilot study. Neural Plasticity, 2016. https:/ / doi.org/10.1155/2016/3136743

Robert, W. (2015). The 3 Golden Rules of Writing A Self-help Book. Retrieved from https:/ / www.standoutbooks.com/how-to-write-a-self-help-book/

Smeltzer, S. C. (2002). Buku Ajar Medikal Bedah Edisi 8 Volume 2. EGC.

Sugiyono. (2011). Metode Penelitian Kuantitatif, Kualitatif dan R \& D. Bandung: Afabeta. 